\title{
ARTICLE OPEN \\ Anion-induced N-doping of naphthalenediimide polymer semiconductor in organic thin-film transistors
}

\author{
Yang Han ${ }^{1,2}$, Zhuping Fei $\mathbb{D}^{1}$, Yen-Hung $\operatorname{Lin}^{2}$, Jaime Martin ${ }^{3}$, Floriana Tuna ${ }^{4}$, Thomas D. Anthopoulos $\mathbb{D}^{2,5}$ and Martin Heeney ${ }^{1}$
}

Molecular doping is an important strategy to improve the charge transport properties of organic semiconductors in various electronic devices. Compared to $\mathrm{p}$-type dopants, the development of $\mathrm{n}$-type dopants is especially challenging due to poor dopant stability against atmospheric conditions. In this article, we report the $n$-doping of the milestone naphthalenediimide-based conjugated polymer P(NDI2OD-T2) in organic thin film transistor devices by soluble anion dopants. The addition of the dopants resulted in the formation of stable radical anions in thin films, as confirmed by EPR spectroscopy. By tuning the dopant concentration via simple solution mixing, the transistor parameters could be readily controlled. Hence the contact resistance between the electrodes and the semiconducting polymer could be significantly reduced, which resulted in the transistor behaviour approaching the desirable gate voltage-independent model. Reduced hysteresis was also observed, thanks to the trap filling by the dopant. Under optimal doping concentrations the channel on-current was increased several fold whilst the on/off ratio was simultaneously increased by around one order of magnitude. Hence doping with soluble organic salts appears to be a promising route to improve the charge transport properties of $n$-type organic semiconductors.

npj Flexible Electronics (2018)2:11; doi:10.1038/s41528-018-0024-2

\section{INTRODUCTION}

Organic semiconductors (OSCs) for organic thin film transistors (OTFTs) have been extensively studied for the development of high-performance next-generation plastic electronics, aiming at flexible, large-area and low-cost devices through processing from solution. ${ }^{1-5}$ Similar to their inorganic counterparts, control of the optoelectronic properties of OSCs by the addition of dopants has proved to be an effective and important strategy in their application and optimisation. ${ }^{6-10}$ When the basic principle of doping is fulfilled, that is, the introduction of dopant molecules results in charge transfer with the host and the generation of additional mobile charge carriers, then the critical properties of devices can become controllable by tuning the amount of dopant. The conductivity of the bulk OSC thin film can be enhanced and the injection barrier between the semiconductor and the electrodes reduced.

The most well understood mechanism for doping is the integer charge transfer mechanism, in which an electron is transferred from the highest occupied molecular orbital (HOMO) of the electron donating semiconductor to the lowest unoccupied molecular orbital (LUMO) of the electron accepting dopant (for $\mathrm{p}$-doping, and vice versa for $\mathrm{n}$-doping). As such there is a general requirement for matching of the ionisation energy of an electron donor and the electron affinity of the electron acceptor. This is challenging for the $n$-doping of OSCs, since to match the LUMO of the host molecules, the HOMO of the dopant has to roughly lie above the range of -3.5 to $-4.5 \mathrm{eV}$ (the LUMO level range of most n-type OSCs), which can make the dopant unstable under atmospheric conditions. ${ }^{6}$ This difficulty in designing stable dopants has somewhat limited the development of $n$-doping of OSCs. ${ }^{11,12}$ Nevertheless a few stable n-type dopants have been reported via mechanisms other than integer charge transfer. ${ }^{13-25}$ For example the addition of $1 \mathrm{H}$-benzimidazole or benzimidazolium salts has succeeded in tuning of the work function of graphene and the conductivity of fullerene derivatives. ${ }^{13,26}$ Mechanistic studies in the solution phase found that the dopant reacted with the fullerene derivative through hydride or hydrogen atom transfer to afford host radical anions, which were responsible for the doping effect. ${ }^{27}$ Comparative studies of the doping efficiency of fullerene-based OSCs with the same dopant in OTFTs also found a significant enhancement in device performance. ${ }^{28}$ However, conjugated polymers, as the major class of solution-processable OSCs, are rarely $\mathrm{n}$-doped in devices, especially OTFTs, ${ }^{14,20,24,29}$ despite the significant improvement it can have on device parameters, e.g., charge carrier mobility, threshold voltage, channel current on/off ratio and contact resistance. Amongst the candidate conjugated n-type polymers for OTFTs, naphthalenediimide (NDI) containing copolymers have shown impressive performance to date. ${ }^{30-35}$ For example the co-

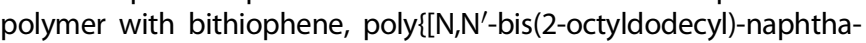
lene-1,4,5,8-bis(dicarboximide)-2,6-diyl]-alt-5,5'-(2,2'-dithiophene)\} (P(NDI2OD-T2)) was one of the first high performance n-type polymers. ${ }^{30,36}$ Further improvements in performance have come from tuning the co-monomer utilised. ${ }^{37,38}$ However, the control of transistor performance by doping of the benchmark polymer itself rather than interface engineering has rarely been mentioned in the literature.

\footnotetext{
${ }^{1}$ Dept. Chemistry and Centre for Plastic Electronics, Imperial College London, Exhibition Rd, London SW7 2AZ, UK; ${ }^{2}$ Dept. Physics and Centre for Plastic Electronics, Imperial College London, Exhibition Rd, London SW7 2AZ, UK; ${ }^{3}$ Dept. of Materials, Imperial College London, Exhibition Rd, London SW7 2AZ, UK; ${ }^{4}$ School of Chemistry and Photon Science

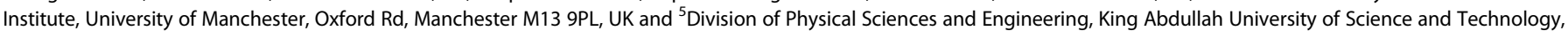
Thuwal 23955-6900, Saudi Arabia

Correspondence: Martin Heeney (m.heeney@imperial.ac.uk)
}

Received: 13 August 2017 Revised: 8 January 2018 Accepted: 6 February 2018

Published online: 16 April 2018 


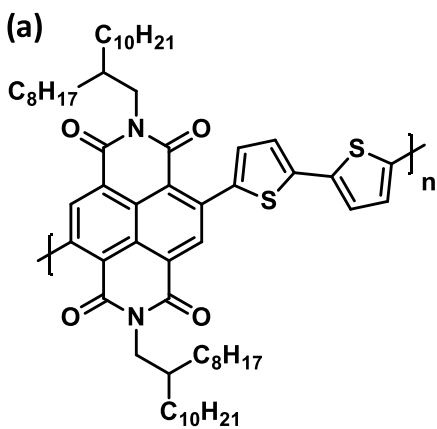

P(NDI2OD-T2)

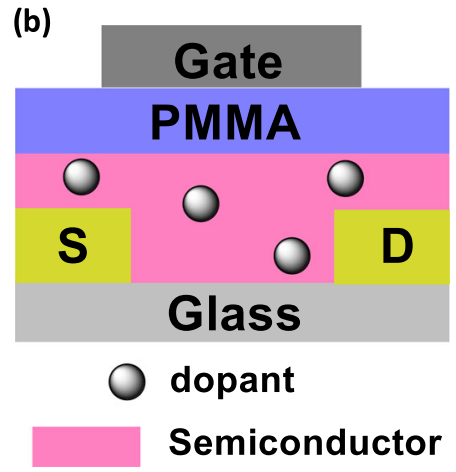

Fig. 1 a Structure of the matrix polymer P(NDI2OD-T2) and dopant TBAF for $n$-doping; $\mathbf{b}$ configuration of top gate/bottom contact OTFT device based on doped polymer

Whilst investigating new $n$-type dopants, we were intrigued by the recent reports by Saha et al. on the interaction between $\pi$ acidic molecular NDI derivatives and a series of soluble Lewis basic anions, in which an electron transfer from the anion to the NDI was proposed to occur. ${ }^{39-41}$ Various functionalised NDI-based molecules were examined in which the electron-deficient nature of the core was modified to tune the strength of the $\pi$-acidity. Saha and co-workers reported that for some NDI-anion pairs, the interaction went beyond the typical non-chromogenic anion- $\pi$ interactions, and that when the energy level of the HOMO of the anion was greater than the LUMO level of the NDI, a thermally induced anion-to-NDI electron transfer was suggested to afford the NDI radical anion in solution. When the HOMO of the anion was lower than the LUMO of the NDI thermal electron transfer was switched off, but photo irradiation could still activate electron transfer from the anion to the NDI. Irrespective of the electron depletion mode of the anion, the corresponding NDI molecule was reduced to the same radical anion product that displayed identical spectroscopic signals. ${ }^{41}$ We note that the exact mechanism of radical anion formation is under debate, particularly with highly electronegative fluoride anions in which direct electron transfer seems thermodynamically unlikely. Nevertheless, Saha's results suggested that soluble anions might be suitable as readily available $n$-type dopants of polymeric NDI derivatives. ${ }^{18,42}$ During the course of this work the use of TBAF to dope fullerene derivatives and some polymer systems has emerged. ${ }^{29,43,44}$ In some cases it appears that nucleophilic addition of the fluoride anion to the fullerene/polymer initially occurs, followed by an electron transfer from the resulting adduct to a neutral polymer chain. ${ }^{43,44}$

To probe the possibility of the above hypothesis, we investigated the $\mathrm{n}$-doping of the benchmark NDI-derivatised polymer P(NDI2OD-T2) with the Lewis basic anion fluoride (in the format of tetrabutylammonium fluoride (TBAF)). We demonstrated that with only a small amount of TBAF dopant, the doping effect was already significant, as determined by OTFT characterisation. Within the optimised dopant concentration range, the injection barrier between the Au source/drain electrodes and semiconductor film could be reduced by around $30 \mathrm{~V}$. The on-current of the device could be increased several fold and $I_{\text {on }} / I_{\text {off }}$ ratio by around one order. Addition of TBAF not only induced the formation of radical species in solution, as previously described in the literature, but it also resulted in the formation of radical products in thin film, as confirmed by electron paramagnetic resonance (EPR) spectroscopy. The generated radical anion was able to control the electrical properties of the matrix NDI polymer by donating additional charges (i.e., electrons). The hysteresis of devices was reduced after doping with fluoride, via trap filling by the dopantcontributed electrons. Though the overall electron mobility of doped P(NDI2OD-T2) was not improved, the devices exhibited a more ideal transistor behaviour compared to the un-doped polymer, with extracted mobility becoming almost independent of gate voltage. Our investigations further confirm the very recent reports on $\mathrm{n}$-doping of polymeric materials by fluoride and hydroxide ions. ${ }^{29}$

\section{RESULTS}

The NDI-based polymer P(NDI2OD-T2) (Fig. 1a) was selected as the model polymer to study $n$-doping with fluoride. The batch of polymer $(\mathrm{Mn}=61 \mathrm{kDa}, \bigoplus=1.66)$ used here was synthesised according to the literature procedure. ${ }^{30}$ The polymer was doped by the addition of a known concentration of TBAF in 1,2dichlorobenzene (DCB) to a solution of the polymer in DCB. The solutions were heated to $140^{\circ} \mathrm{C}$ under stirring prior to deposition by spin coating. The resulting films were then thermally annealed at $200^{\circ} \mathrm{C}$ for $30 \mathrm{~min}$ before further characterisation.

\section{EPR spectroscopy}

According to the reports by Saha et al., the strong Lewis basic fluoride anion interacted with various NDI molecules by a thermally induced electron-transfer mechanism to form $\mathrm{NDI}^{-}$ radical anions in aprotic solvents. ${ }^{41}$ Since the Lewis acidity of the NDIs was not the reported limitation for the electron transfer in this process, we predicted that thermally induced electron-transfer might also occur with the NDI units of P(NDI2OD-T2), leading to a n-doping effect.

To confirm the presence of radical anions in the solid state, EPR spectroscopy was performed upon films formed from solutions doped with varying concentrations of TBAF, as shown in Fig. 2 a. The molar equivalent of fluoride dopant TBAF was calculated with respect to the mass of repeat units of the polymer (i.e., NDI-T2). The un-doped P(NDI2OD-T2) is diamagnetic and no EPR signals were observed. However, upon addition of TBAF clear signals of paramagnetic radical species were observed. The signal intensity rapidly saturated with increasing microwave power (Figure S1), as expected for organic radicals. With small amounts (0.025 eq.) of TBAF dopant the EPR signal showed a five-line pattern centred around $g=2.005$, which is most likely due to hyperfine splitting by the two ${ }^{14} \mathrm{~N}$ nuclei of the $\mathrm{NDI}^{-}$radical anion. Here the ${ }^{14} \mathrm{~N}$ hyperfine coupling is much larger (>14 G) and dominant over ${ }^{1} \mathrm{H}$ couplings, which remain unresolved. We note that the spectra for the doped film is significantly different to that observed in solution for radical anion of $\mathrm{NDI}^{45}$ and the unequal coupling observed likely arises from different paramagnetic environments expected in the solid state. When larger amounts ( 0.25 and 1 eq.) of dopant were added, the hyperfine splitting structure disappeared and the signals narrows with dopant concentration. We attribute this narrowing to a fast spin exchange as the 
(a)

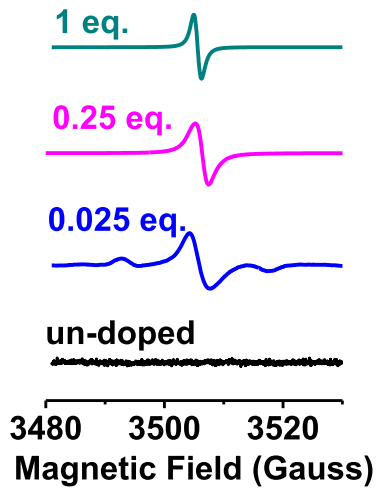

(b)

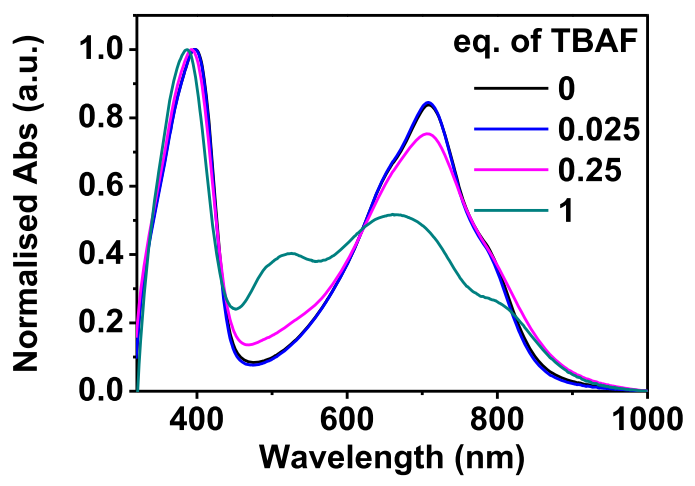

Fig. 2 a EPR and $\mathbf{b}$ UV-Vis absorption spectra of un-doped and doped polymer films with representative dopant concentrations
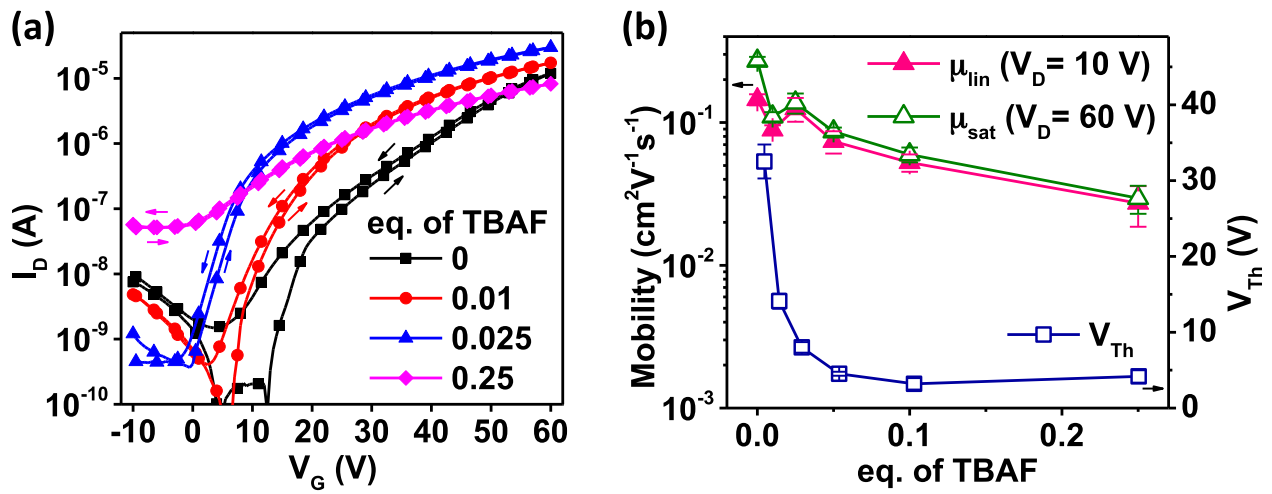

Fig. 3 a Representative plots of the transfer characteristics of transistors based on un-doped and doped $P(N D I 2 O D-T 2)$ at $V_{D}=60 V$; $\mathbf{b}$ dependence of saturation (hollow triangle) and linear (filled triangle) mobility, and threshold voltage (hollow square) on doping equivalents of TBAF. The central symbol denotes the average level, and the upper and lower limit indicates standard deviation

concentration of radicals increases, in agreement with other studies. ${ }^{46}$ Interestingly we also found that the signal intensity increases with dopant concentration. Double integration of the EPR spectra recorded under identical conditions for films doped with $0.025,0.25$ and 1 eq. demonstrate a systematic increase with dopant density (Figure S2). Whilst these measurements are not quantitative, due to the difficulties associated with preparing identical film samples, they nevertheless suggest that TBAF concentration can be used to control the doping density.

\section{UV-Vis absorption spectroscopy}

We also attempted to detect the formation of the radical species by thin-film UV-Vis spectroscopy (Fig. 2b). However, at the lowest dopant concentration ( 0.025 eq.), no obvious new peak could be observed, likely due to the small amount of radical anion formed in addition to overlapping peaks from the host polymer. The absorption spectra of this film was almost identical to that of the un-doped P(NDI2OD-T2) polymer, showing two main absorption bands at 398 and $709 \mathrm{~nm}$ in film, similar to the reported data. ${ }^{30}$ When the amount of fluoride dopant was increased to 0.25 eq. the two absorption bands were slightly blue shifted, to 394 and $706 \mathrm{~nm}$ respectively, with the relative intensity of the lower energy region reduced. A new absorption feature started to evolve between these two main bands. This became more pronounced upon increasing the fluoride dopant density to 1 eq., with a clear peak observable at $543 \mathrm{~nm}$, which can be ascribed to the existence of $\mathrm{NDI}^{-}{ }^{-}$by addition of TBAF to P(NDI2OD-T2). ${ }^{41}$ In addition the longer wavelength peaks shift and change in intensity. The changes are similar to those observed upon electrochemical reduction of P(NDI2OD-T2). ${ }^{47}$

\section{OTFT characterisation}

To examine the doping effect of TBAF on the electrical properties of $\mathrm{P}(\mathrm{NDI} 2 \mathrm{OD}-\mathrm{T2})$, un-doped and doped polymer layers were investigated in OTFT devices with a top-gate, bottom-contact (TG/ $\mathrm{BC}$ ) configuration fabricated via solution processing. The transfer characteristics of a set of transistors based on un-doped and doped polymer with representative equivalents of dopant are shown in Fig. 3. The un-doped polymer exhibited typical n-type behaviour, with field-effect electron mobility of $0.15( \pm 0.013)$ in the linear and $0.27( \pm 0.018) \mathrm{cm}^{2} \mathrm{~V}^{-1} \mathrm{~s}^{-1}$ in the saturation regime, channel current on/off ratio in the range of $10^{3}-10^{4}$, and threshold voltage $\left(\mathrm{V}_{\mathrm{Th}}\right)$ of $32.5( \pm 2.2) \mathrm{V}$. The high value of $\mathrm{V}_{\mathrm{Th}}$ indicates a relatively large electron injection barrier, which is probably due to the difference between the work function of $\mathrm{Au}$ electrodes $(\sim-5.0 \mathrm{eV})$ and LUMO of P(NDI2OD-T2) $(\sim-4.0 \mathrm{eV})$. The use of $\mathrm{Au}$ electrodes also results in some hole injection into the HOMO of P(NDI2OD-T2) $(\sim-5.6 \mathrm{eV})$. Noticeable hole transport can be observed in the transfer scans. The hysteresis observed between the forward and reverse scan is likely due to the presence of charge carrier traps.

As shown in Fig. 3a, the addition of TBAF has an obvious doping effect on transistor device performance. The key parameters of transistors based on doped P(NDI2OD-T2) with a full series of dopant concentrations are summarised in Table S1 and their transfer and output characteristics are shown in Figure S3. Small amounts (0.01 eq.) of dopant resulted in an increase in on-current and a reduction in hysteresis. Extraction of the threshold voltage (Fig. 3b) shows a very significant shift of $18 \mathrm{~V}$ towards the negative direction. These doping effects at very low dopant concentration can be attributed to the trap filling effect. ${ }^{48,49}$ Hole transport is still observable at this low dopant concentration. The doping 
efficiency was further enhanced when the amount of TBAF was increased to 0.025 eq., at which concentration the transistor reached its highest on-current level. The off-current remained low at this concentration and the channel on/off ratio was increased by around one order accordingly. The threshold voltage was further reduced to $8.0( \pm 0.7) \mathrm{V}$, and the hysteresis between the forward and reverse scan became negligible, which we ascribe to trap filling by the additional dopant-contributed charges generated by the introduction of the dopant. Moreover, the hole transport was completely suppressed.

As the TBAF concentration was further increased from 0.025 eq., the on channel current started to drop and the off-current increased, resulting in lower on/off ratios compared to the undoped (pristine) or 0.01 and 0.025 eq. TBAF doped polymer devices. The increase in off-current is probably due to the increase in bulk conductivity of the semiconductor layer by doping, which makes it progressively more difficult to switch off the transistor until the gate bias is significantly negative. ${ }^{28}$ As shown in Fig. 3b, the threshold voltage of the devices continues to decrease as the dopant concentration was increased, until reaching $3-4 \mathrm{~V}$ at a dopant concentration of 0.25 eq. Higher levels of doping resulted in devices becoming too conductive and therefore unable to switch off.

An interesting effect of the doping process was on the gate voltage dependence of the extracted electron mobility values. As shown in Figure S4c, the linear and saturation mobility of undoped devices shows a pronounced gate voltage dependence, indicative of significant contact resistance. As a result the highest mobility could only be obtained at a certain level of gate bias, a phenomenon which has been intensively discussed recently and attributed to parasitic contact resistance. ${ }^{50-52}$ In addition, an apparent mismatch between the linear and saturation mobility was also observed for the un-doped device, which was attributed to the contact resistance between the electrode and semiconductor as discussed above. ${ }^{53,54}$ When the TBAF dopant was added, the difference between the saturation and linear mobility was reduced, and the mobility calculation from first derivative of the linear regime transfer curve and first derivative of the square root of the saturation regime transfer curve becomes more gate voltage-independent (Figure S4f). We attribute this to the reduction in contact resistance, which was as high as $4.52 \mathrm{M} \Omega \cdot \mathrm{cm}$ for un-doped polymer, and gradually decreased by two orders of magnitude by doping with different concentrations of TBAF (Figure S5 and Table S1).

The doping effect of TBAF is a somewhat unusual one, and given recent reports on the use of tertiary amines as n-type dopants, ${ }^{23}$ we were interested if the presence of tributylamine (TBA) may have caused the doping effect. TBA may either have been present as an impurity in the commercial TBAF, or may arise from Hofmann type eliminations occurring during thermal annealing. ${ }^{23}$ Therefore we investigated the device performance of films formed from solutions with 0.025 eq. of TBA (Figure S6). No obvious doping effect was observed, with similar on-current and threshold voltage compared to the un-doped device, suggesting that the addition of TBA had no impact on injection improvement. Moreover, the use of different anions $\left(\mathrm{Cl}^{-}, \mathrm{Br}^{-}, \mathrm{I}^{-}\right)$as tetrabutylammonium salts resulted in similar doping effects to TBAF. The device characteristics are shown in Figure S6 and summarised in Table S2. The mismatch between linear and saturation mobility was again reduced, and the injection barrier, expressed by the threshold voltage, was also reduced to a similar range to that of the TBAF doped devices. It should be noted that $\mathrm{Cl}^{-}, \mathrm{Br}^{-}$and $\mathrm{I}^{-}$are much less basic than $\mathrm{F}^{-}$and unlikely to trigger the Hofmann elimination reaction, suggesting the doping effect in this study is rather related to the anion- $\pi$ electron transfer.

The generation of radical anions is expected to impact the concentration of the charge trap states and hence influences the performance of the resulting transistors. To study this effect we

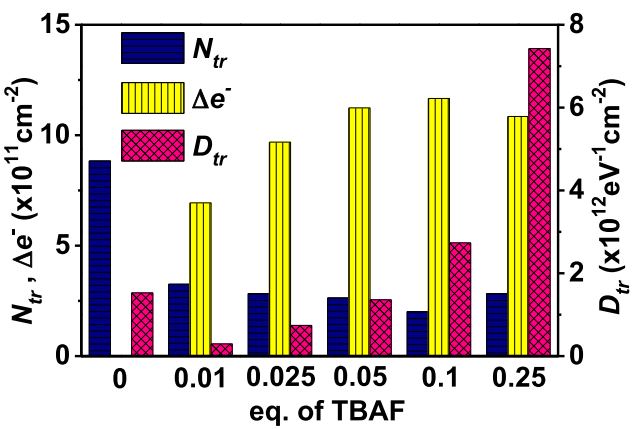

Fig. 4 Additional free electrons generated by addition of TBAF $\left(\Delta e^{+}\right)$, interface trap density $\left(N_{\mathrm{tr}}\right)$ and trap concentration per energy unit $\left(D_{\mathrm{tr}}\right)$ of transistors based on un-doped and doped P(NDI2OD-T2) as a function of equivalents of TBAF

calculated the interface trap density $\left(N_{\text {tr }}\right)$ and trap concentration $\left(D_{\text {tr }}\right.$ per unit area and unit energy) using the following equations: ${ }^{55,56}$

$N_{\mathrm{tr}}=\frac{C_{i}}{e}\left|V_{\mathrm{Th}}-V_{\mathrm{on}}\right|$

and

$D_{\mathrm{tr}}=\frac{C_{i}}{e^{2}}\left(\frac{e S}{k \ln (10)}-1\right)$

where $e$ is the elementary charge, $C_{\mathrm{i}}$ the geometric capacitance of gate dielectric, $V_{\text {on }}$ the onset voltage, $k$ the Boltzmann constant, $T$ the measuring temperature and $S$ the subthreshold swing. The calculated trap density and trap concentration are shown in Fig. 4. The interface trap density $\left(N_{\mathrm{tr}}\right)$ was found to decrease upon doping with small amounts of TBAF, and continuingly reduced as the dopant concentration was increased to 0.1 eq., followed by a slight increase at 0.25 eq. The evolution of interface trap density is consistent with the change in threshold voltage as a function of dopant concentration. The trap concentration $\left(D_{\mathrm{tr}}\right)$, on the other hand, is reduced by the addition of 0.01 eq. of TBAF dopant, but further increasing TBAF concentration results in an increase in trap concentration, with higher levels of traps at concentrations higher than 0.05 eq. compared to the un-doped transistor. The latter observation is attributed to the structural defects/energetic disorder induced in the bulk of the polymer by the dopant at high concentrations.

Besides modulating the density of trap states, efficient doping at appropriate levels is also known to introduce dopantcontributed charges (electrons) which under certain circumstances can help to enhance the charge carrier transport. The amount of dopant-contributed electrons induced by TBAF doping was also calculated from the operating characteristics of the transistors using: ${ }^{28,57}$

$\Delta h=\frac{C_{i}}{e}\left|V_{\mathrm{Th}(\text { doped })}-V_{\mathrm{Th}(\text { un }- \text { doped })}\right|$.

As shown in Fig. 4, the number of dopant-contributed electrons generated by adding TBAF to P(NDI2OD-T2) is of the order of $(6-12) \times 10^{11} \mathrm{~cm}^{-2}$, with a slight yet clear trend that increases with higher dopant concentration. The evolution of extra dopantcontributed electrons is in agreement with that of interface trap density, and also mirrors the threshold voltage shift of the transistor devices. Overall, the introduction of the dopant fills charge carrier traps at the interface rather than deep in the semiconductor layer. The injection barrier and contact resistance are accordingly reduced. 

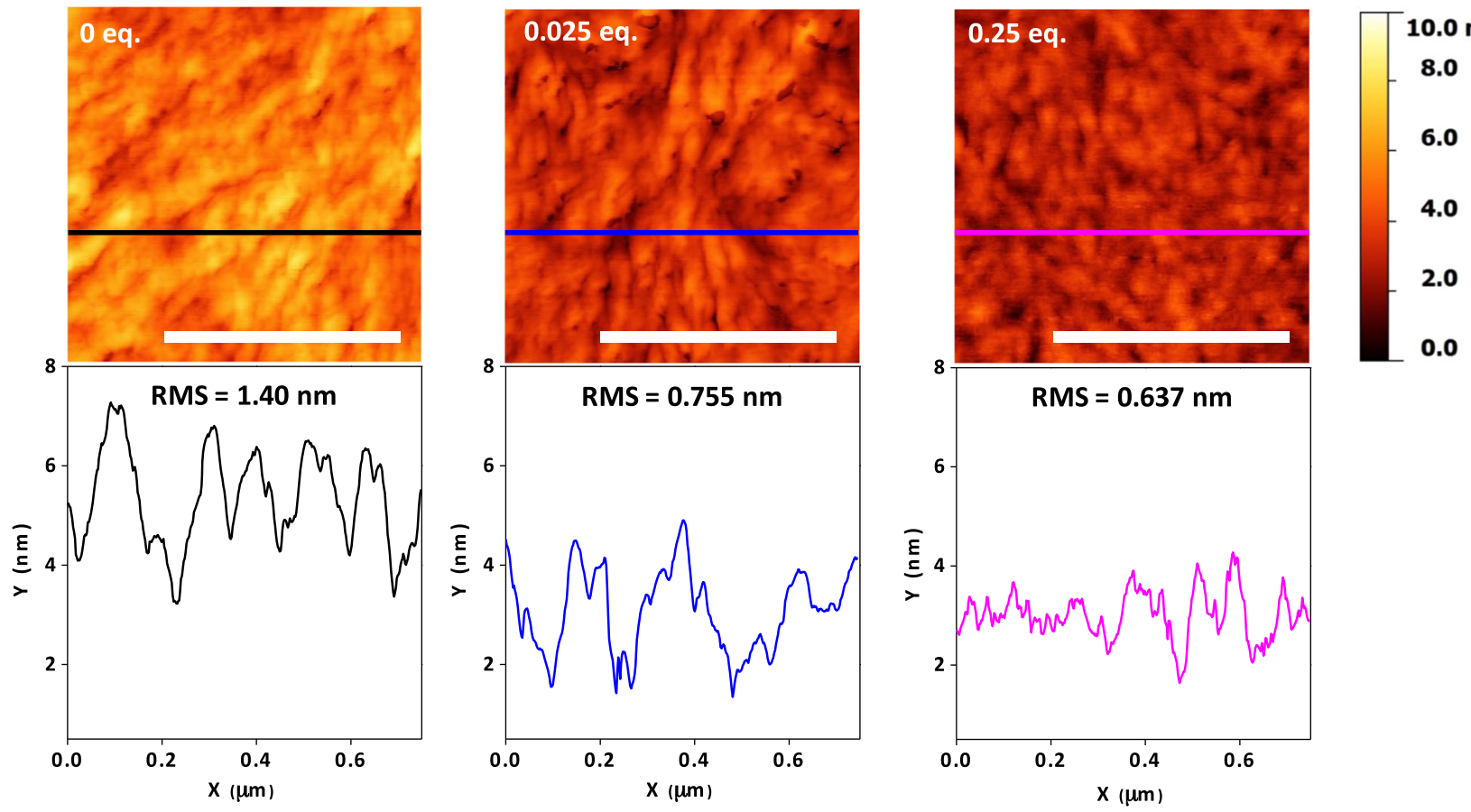

Fig. 5 AFM topography images (top) and height profiles (bottom) of un-doped and doped P(NDI2OD-T2) films with different equivalents of TBAF (Scale bar: $500 \mathrm{~nm}$ )

Film morphology

Atomic force microscopy (AFM) was employed to study the effects of doping on film surface morphology. Un-doped and doped $P$ (NDI2OD-T2) films were spun-cast on glass substrates and annealed following identical procedures to those for the deposition of the transistor semiconductor layer. As shown in Fig. 5, the un-doped P(NDI2OD-T2) affords a continuous film with an entangled fibrillar microstructure, similar to the morphology of spun-cast films previously reported. ${ }^{30}$ The root mean square surface roughness (RMS) of the un-doped film was $1.40 \mathrm{~nm}$. In contrast to reports where dopant phase-segregated from the host, ${ }^{58,59}$ TBAF doped P(NDI2OD-T2) showed no obvious phaseseparated domains formed by the dopant. Films doped with 0.025 and 0.25 eq. TBAF display similar morphology to that of the undoped one, with a RMS roughness of 0.755 and $0.637 \mathrm{~nm}$, respectively. The slight decrease in surface roughness is probably due to formation of a less pronounced semicrystalline structure by the insertion of dopant to the polymer matrix.

To probe the packing mode of molecular chains, wide-angle $X$ ray diffraction (XRD) was also carried out on films before and after doping (Figure S7). The un-doped and doped films displayed very similar diffraction patterns. Diffraction peaks at $2 \theta=3.62^{\circ}$ and $7.20^{\circ}$ corresponding to d-spacing of $24.38 \mathrm{~nm}$ and $12.26 \mathrm{~nm}$ were observed in both cases, which can be attributed to the first (100) and second (200) order lamellar packing of the conjugated polymer backbones. A diffraction peak at $2 \theta=22.84^{\circ}(d=$ $3.89 \mathrm{~nm}$ ) was also observed and attributed to the $\pi-\pi$ spacing between two adjacent backbones. Hence the addition of the dopant did not obviously hinder packing of the polymer film or significantly influence its surface topology.

\section{DISCUSSION}

Since n-type dopants designed according to the traditional integer charge transfer principle often encounter air stability problems due to their small ionisation potentials, the development of new n-doping motifs is highly desirable to improve the charge transport properties of electron transport semiconductors.
Furthermore the controlled doping of polymeric systems can be especially challenging from the solution phase, since the polymers are prone to aggregate and precipitate during the doping process. Although polymeric films can be doped via the vapour phase after film formation, the direct fabrication of doped films from the solution phase removes additional processing steps and is attractive for the development of printed devices. Here we have shown that the addition of soluble tetrabutylammonium salts is an attractive approach to the formation of doped devices that utilises readily available dopants and enables full solution processing without aggregation problems. Although the exact mechanism of doping is not yet resolved, we have demonstrated that the addition of a soluble fluoride source (TBAF) to a naphthalene diimide containing conjugated polymer had a significant impact on the electrical properties of the polymer via the formation of radical anions. Radical anion formation was confirmed by EPR and UV-Vis absorption studies of thin films of the doped polymer. By adjusting the concentration of the dopant via solution processing, the performance of OTFTs was readily modulated. A combination of trap filling and the introduction of dopant-contributed electrons by $n$-doping dramatically reduced the electron injection barrier and contact resistance between the metal contacts and the semiconductor, resulting in improved device performance. Notably doped transistors showed near ideal transistor behaviour with close to gate voltage independent mobility values. The above $n$ doping method was also extended to other soluble anions such as $\mathrm{Cl}^{-}, \mathrm{Br}^{-}$and $\mathrm{I}^{-}$, further demonstrating the utility of this approach for $n$-doping.

\section{METHODS}

General

All chemicals and solvents were purchased from Sigma Aldrich and used as received unless otherwise specified. Gel permeation chromatography (GPC) was carried out on Agilent Technologies 1200 series GPC. Numberaverage $(\mathrm{Mn})$ and weight-average $(\mathrm{Mw})$ were determined by running in chlorobenzene at $80^{\circ} \mathrm{C}$, using two $\mathrm{PL}$ mixed $\mathrm{B}$ columns in series, and calibrated against narrow polydispersity polystyrene standards. UV-Vis spectra were recorded on a UV-1601 Shimadzu UV-Vis spectrometer. EPR 
measurements were performed at room temperatures on a Bruker ElexSys E580 spectrometer operating at X-band frequency (ca. $9.85 \mathrm{GHz}$ ) and the observed power was $18 \mathrm{~dB}$ under $10 \mathrm{~mW}$. Films were prepared by drop casting the un-doped or doped solutions on glass substrates under nitrogen. Films were briefly exposed to air during transfer to a $4 \mathrm{~mm}$ quartz EPR tube which was filled with argon. Wide-angle XRD (PANalytical X'Pert Pro MPD, Cu Ka radiation) was carried out to probe the molecular packing, and $\theta / 2 \theta$ scans were performed at room temperature to the films drop cast from the corresponding mixture solutions onto silicon substrates. Tapping mode AFM was carried out with a Picoscan PicoSPM LE scanning probe. Samples were prepared by spin coating polymer solutions on plain glass and thermal annealing treatment afterwards at $200^{\circ} \mathrm{C}$, following the identical procedures for fabrication of OTFT devices, except that dielectric and gate electrodes were not deposited.

Synthesis and characterisation of poly $\left\{\left[\mathrm{N}, \mathrm{N}^{\prime}\right.\right.$-bis(2-octyldodecyl)naphthalene-1,4,5,8-bis(dicarboximide)-2,6-diyl]-alt-5,5'-(2,2'dithio-phene)\} (P(NDI2OD-T2))

$\mathrm{P}\left(\mathrm{NDI}\right.$ 2OD-T2) was synthesised according to the literature procedure. ${ }^{30}$ The number average molecular weight and polydispersity index were determined by GPC against narrow polydispersity polystyrene standards to be $61 \mathrm{kDa}$ and $1.66 \mathrm{kDa}$, respectively.

\section{Preparation of complex solutions}

In a nitrogen glove box, stock solutions of polymer and dopants were prepared, respectively, prior to mixing, by dissolving $\mathrm{P}(\mathrm{NDI} 2 \mathrm{OD}-\mathrm{T} 2)$, tetrabutylammonium fluoride trihydrate $\left(\mathrm{TBAF} \cdot 3 \mathrm{H}_{2} \mathrm{O}\right)$, tetrabutylammonium chloride, tetrabutylammonium bromide, tetrabutylammonium iodide, and TBA in DCB to reach a concentration of $10 \mathrm{mM}$. The polymer concentration and molar equivalents of dopant were determined with respect to the mass of repeat units of the polymer. Each solution with the required amount of dopant was prepared by adding the corresponding volume of dopant solution to $100 \mu \mathrm{L}$ of the polymer solution. Additional DCB was added to each of the above mixture solutions if necessary to dilute to a volume of $150 \mu \mathrm{L}$. The final concentration of polymer was kept constant $(6.67 \mathrm{mM})$. The solutions were stirred at $140^{\circ} \mathrm{C}$ for $10 \mathrm{~min}$ and allowed to cool to room temperature before spin coating.

\section{Fabrication of OTFT devices}

The top gate/bottom contact (TG/BC) configuration was employed to fabricate transistor devices based on un-doped and doped polymers. Bottom contact substrates were prepared by thermal evaporation of $\mathrm{Au}$ $(40 \mathrm{~nm})$ onto glass substrates through a shadow mask. The above mentioned solutions were then spun-cast at $2000 \mathrm{rpm}$ for $60 \mathrm{~s}$ onto prepatterned substrates. The obtained semiconductor films were thermally annealed at $200^{\circ} \mathrm{C}$ for $30 \mathrm{~min}$. Poly(methyl methacrylate) (PMMA, average $\mathrm{Mw} \sim 120 \mathrm{kDa}$ ) $80 \mathrm{mg} / \mathrm{mL}$ solution in $\mathrm{n}$-butyl acetate was then spin-coated on top, followed by annealing at $80^{\circ} \mathrm{C}$ for $2 \mathrm{~h}$ to give the dielectric layer. $\mathrm{Al}$ $(40 \mathrm{~nm})$ gate electrodes were evaporated on top of dielectric through shadow mask to complete the TG/BC transistor devices. All processing and measurements were performed in a nitrogen glove box. Transistor characterisation was carried out under nitrogen using a Keithley 4200 parameter analyser. The saturation mobility was extracted from the slope of $I_{D}^{1 / 2}$ vs. $V_{G}$ :

$\mu_{\mathrm{sat}}=\frac{2 L}{W C_{\mathrm{i}}}\left(\frac{\partial \sqrt{\mathrm{D} \mathrm{sat}}}{\partial V_{\mathrm{G}}}\right)^{2}$

and linear mobility was extracted from the equation below:

$\mu_{\operatorname{lin}}=\frac{L}{W C_{\mathrm{i}} V_{\mathrm{D}}}\left(\frac{\partial I_{\mathrm{Dlin}}}{\partial V_{\mathrm{G}}}\right)$

The channel width and length of the final transistors were $1 \mathrm{~mm}$ and 40 $\mu \mathrm{m}$, respectively, unless otherwise specified.

\section{Data availability}

The raw data that support the findings of this study are available in figshare with the identifier https://doi.org/10.6084/m9.figshare.5766399.

\section{ACKNOWLEDGEMENTS}

We thank the National EPR Facility and Service Centre in The University of Manchester for experimental and data analysis support on EPR spectroscopy. This work was financially supported by the British Council (grant number 173601536).

\section{AUTHOR CONTRIBUTIONS}

Y.H., Z.F., T.D.A. and M.H. designed experiments. Y.H. fabricated and measured OTFT devices, carried out UV-Vis and AFM spectroscopy. Z.F. synthesised P(NDI2OD-T2) polymer. Y.-H.L. carried out Kelvin Probe experiment and analysed data. J.M. performed X-ray diffraction and analysed data. F.T. performed EPR measurements and analysed the data. T.D.A. and M.H. supervised the overall project. All authors contributed to analysis and writing.

\section{ADDITIONAL INFORMATION}

Supplementary information accompanies the paper on the npj Flexible Electronics website (https://doi.org/10.1038/s41528-018-0024-2).

Competing interests: The authors declare no competing interests.

Publisher's note: Springer Nature remains neutral with regard to jurisdictional claims in published maps and institutional affiliations.

\section{REFERENCES}

1. Baeg, K. J., Caironi, M. \& Noh, Y. Y. Toward printed integrated circuits based on unipolar or ambipolar polymer semiconductors. Adv. Mater. 25, 4210-4244 (2013).

2. Gsänger, M., Bialas, D., Huang, L., Stolte, M. \& Würthner, F. Organic semiconductors based on dyes and color pigments. Adv. Mater. 28, 3615-3645 (2016).

3. Horowitz, G. Organic field-effect transistors. Adv. Mater. 10, 365-377 (1998).

4. Zaumseil, J. \& Sirringhaus, H. Electron and ambipolar transport in organic fieldeffect transistors. Chem. Rev. 107, 1296-1323 (2007).

5. Newman, C. R. et al. Introduction to organic thin film transistors and design of nchannel organic semiconductors. Chem. Mater. 16, 4436-4451 (2004).

6. Walzer, K., Maennig, B., Pfeiffer, M. \& Leo, K. Highly efficient organic devices based on electrically doped transport layers. Chem. Rev. 107, 1233-1271 (2007).

7. Lüssem, B., Riede, M. \& Leo, K. Doping of organic semiconductors. Phys. Status Solidi A Appl. Mater. Sci. 210, 9-43 (2013).

8. Yoo, S.-J. \& Kim, J.-J. Charge transport in electrically doped amorphous organic semiconductors. Macromol. Rapid Commun. 36, 984-1000 (2015).

9. Salzmann, I., Heimel, G., Oehzelt, M., Winkler, S. \& Koch, N. Molecular electrical doping of organic semiconductors: fundamental mechanisms and emerging dopant design rules. Acc. Chem. Res. 49, 370-378 (2016).

10. Lüssem, B. et al. Doped organic transistors. Chem. Rev. 116, 13714-13751 (2016).

11. Chan, C. K., Kahn, A., Zhang, Q., Barlow, S. \& Marder, S. R. Incorporation of cobaltocene as an $\mathrm{n}$-dopant in organic molecular films. J. Appl. Phys. 102, 14906 (2007).

12. Chan, C. K., Zhao, W., Barlow, S., Marder, S. \& Kahn, A. Decamethylcobaltocene as an efficient $\mathrm{n}$-dopant in organic electronic materials and devices. Org. Electron. Phys., Mater. Appl. 9, 575-581 (2008).

13. Wei, P., Oh, J. H., Dong, G. \& Bao, Z. Use of a $1 \mathrm{H}$-benzoimidazole derivative as an $\mathrm{n}$-type dopant and to enable air-stable solution-processed n-channel organic thin-film transistors. J. Am. Chem. Soc. 132, 8852-8853 (2010).

14. Cho, N. et al. In-situ crosslinking and n-doping of semiconducting polymers and their application as efficient electron-transporting materials in inverted polymer solar cells. Adv. Energy Mater. 1, 1148-1153 (2011).

15. Lu, M., Nicolai, H. T., Wetzelaer, G. J. A. H. \& Blom, P. W. M. N-type doping of poly (p-phenylene vinylene) with air-stable dopants. Appl. Phys. Lett. 99, 173302 (2011).

16. Guo, S. et al. N-doping of organic electronic materials using air-stable organometallics. Adv. Mater. 24, 699-703 (2012).

17. Wei, P. et al. 2-(2-Methoxyphenyl)-1,3-dimethyl-1 H-benzoimidazol-3-ium iodide as a new air-stable n-type dopant for vacuum-processed organic semiconductor thin films. J. Am. Chem. Soc. 134, 3999-4002 (2012).

18. Li, C. Z. et al. Doping of fullerenes via anion-induced electron transfer and its implication for surfactant facilitated high performance polymer solar cells. Adv. Mater. 25, 4425-4430 (2013).

19. Fabiano, S. et al. Poly(ethylene imine) Impurities Induce $n$-doping Reaction in Organic (Semi)Conductors. Adv. Mater. 26, 6000-6006 (2014).

20. Sun, B. et al. Polyethylenimine (PEI) as an effective dopant to conveniently convert ambipolar and p-type polymers into unipolar $\mathrm{n}$-type polymers. ACS Appl. Mater. Interfaces 7, 18662-18671 (2015). 
21. Higgins, A., Mohapatra, S. K., Barlow, S., Marder, S. R. \& Kahn, A. Dopant controlled trap-filling and conductivity enhancement in an electron-transport polymer. Appl. Phys. Lett. 106, 163301 (2015)

22. Voortman, T. P., Bartesaghi, D., Koster, L. J. A. \& Chiechi, R. C. Cross-conjugated ndopable aromatic polyketone. Macromolecules 48, 7007-7014 (2015).

23. Russ, B. et al. Tethered tertiary amines as solid-state n-type dopants for solutionprocessable organic semiconductors. Chem. Sci. 7, 1914-1919 (2016).

24. Luo, H. et al. Remarkable enhancement of charge carrier mobility of conjugated polymer field-effect transistors upon incorporating an ionic additive. Sci. Adv. 2, E1600076 (2016).

25. Arulkashmir, A., Jain, B., John, J. C., Roy, K. \& Krishnamoorthy, K. Chemically doped perylene diimide lamellae based field effect transistor with low operating voltage and high charge carrier mobility. Chem. Commun. 50, 326-328 (2014).

26. Wei, P. et al. Tuning the dirac point in cvd-grown graphene through solution processed $\mathrm{n}$ type doping with 2 (2-methoxyphenyl)-1,3-dimethyl-2,3-dihydro $1 \mathrm{H}$ benzoimidazole. Nano. Lett. 13, 1890 (2013).

27. Naab, B. D. et al Mechanistic study on the solution-phase n-doping of 1,3dimethyl-2-aryl-2,3-dihydro-1H-benzoimidazole derivatives. J. Am. Chem. Soc. 135, 15018-15025 (2013)

28. Rossbauer, S., Müller, C. \& Anthopoulos, T. D. Comparative study of the N-type doping efficiency in solution-processed fullerenes and fullerene derivatives. Adv. Funct. Mater. 24, 7116-7124 (2014).

29. Kim, J. et al. Systematic study of widely applicable N-doping strategy for highperformance solution-processed field-effect transistors. Adv. Funct. Mater. 26 7886-7894 (2016).

30. Yan, $\mathrm{H}$. et al. A high-mobility electron-transporting polymer for printed transistors. Nature 457, 679-686 (2009).

31. Matsidik, R., Komber, H., Luzio, A., Caironi, M. \& Sommer, M. Defect-free naphthalene diimide bithiophene copolymers with controlled molar mass and high performance via direct arylation polycondensation. J. Am. Chem. Soc. 137, 6705 (2015).

32. Kim, R. et al. High-mobility air-stable naphthalene diimide-based copolymer containing extended $\pi$-conjugation for $\mathrm{n}$-channel organic field effect transistors. Adv. Funct. Mater. 23, 5719-5727 (2013).

33. Guo, X., Facchetti, A. \& Marks, T. J. Imide- and Amide-functionalized polymer semiconductors. Chem. Rev. 114, 8943-9021 (2014).

34. Liu, Z. et al. New organic semiconductors with imide/amide-containing molecular systems. Adv. Mater. 26, 6965-6977 (2014).

35. Zhan, X. et al. Rylene and related diimides for organic electronics. Adv. Mater. 23, 268-284 (2011).

36. Kim, N. K. et al. Solution-processed barium salts as charge injection layers for high performance N-channel organic field-effect transistors. ACS Appl. Mater. Interfaces 6, 9614-9621 (2014).

37. Chen, $\mathrm{H}$. et al. Naphthalenediimide-based copolymers incorporating vinyl- linkages for high-performance ambipolar field-effect transistors and complementary-like inverters under air. Chem. Mater. 25, 3589-3596 (2013).

38. Kim, J. H. et al. New quinoxaline derivatives as accepting units in donor-acceptor type low-band gap polymers for organic photovoltaic cells. J. Polym. Sci. Part A Polym. Chem. 51, 4136-4149 (2013).

39. Guha, S. \& Saha, S. Fluoride ion sensing by an anion- $\pi$ interaction. J. Am. Chem. Soc. 132, 17674-17677 (2010).

40. Guha, S. et al. Electronically regulated thermally and light-gated electron transfer from anions to naphthalenediimides. J. Am. Chem. Soc. 133, 15256-15259 (2011).

41. Guha, S., Goodson, F. S., Corson, L. J. \& Saha, S. Boundaries of anion/naphthalenediimide interactions: from anion $-\pi$ interactions to anion-induced chargetransfer and electron-transfer phenomena. J. Am. Chem. Soc. 134, 13679-13691 (2012).

42. Lafleur-Lambert, A., Giguère, J.-B. \& Morin, J.-F. Conjugated polymers based on 4,10-bis(thiophen-2-yl)anthanthrone: synthesis, characterization, and fluoridepromoted photoinduced electron transfer. Macromolecules 48, 8376-8381 (2015).
43. Zhao, X. et al. High conductivity and electron-transfer validation in an n-type fluoride-anion-doped polymer for thermoelectrics in air. Adv. Mater. 29, 1606928 (2017)

44. Weber, C. D., Bradley, C. \& Lonergan, M. C. Solution phase $n$-doping of $C_{60}$ and PCBM using tetrabutylammonium fluoride. J. Mater. Chem. A 2, 303-307 (2014).

45. Bhosale, S. V., Jani, C. H. \& Langford, S. J. Chemistry of naphthalene diimides. Chem. Soc. Rev. 37, 33-342 (2008).

46. Zaka, M. et al. Exchange interactions of spin-active metallofullerenes in solid-state carbon networks. Phys. Rev. B Condens. Matter Mater. Phys. 81, 1-5 (2010).

47. Trefz, D. et al. Electrochemical investigations of the N-type semiconducting polymer $\mathrm{P}(\mathrm{NDI} 2 \mathrm{OD}-\mathrm{T} 2)$ and its monomer: new insights in the reduction behavior. J. Phys. Chem. C 119, 22760-22771 (2015).

48. Tietze, M. L., Burtone, L., Riede, M., Lüssem, B. \& Leo, K. Fermi level shift and doping efficiency in p-doped small molecule organic semiconductors: a photoelectron spectroscopy and theoretical study. Phys. Rev. B Condens. Matter Mater. Phys. 86, 35320 (2012).

49. Olthof, S. et al. Ultralow doping in organic semiconductors: evidence of trap filling. Phys. Rev. Lett. 109, 176601 (2012).

50. Sirringhaus, H. 25th anniversary article: organic field-effect transistors: the path beyond amorphous silicon. Adv. Mater. 26, 1319-1335 (2014).

51. Mcculloch, I., Salleo, A. \& Chabinyc, M. Avoid the kinks when measuring mobility Science 352, 1521-1522 (2016).

52. Bittle, E. G., Basham, J. I., Jackson, T. N., Jurchescu, O. D. \& Gundlach, D. J. Mobility overestimation due to gated contacts in organic field-effect transistors. Nat. Commun. 7, 10908 (2016).

53. Zaumseil, J., Baldwin, K. W. \& Rogers, J. A. Contact resistance in organic transistors that use source and drain electrodes formed by soft contact lamination. J. Appl. Phys. 93, 6117-6124 (2003).

54. Lee, B. H., Bazan, G. C. \& Heeger, A. J. Doping-induced carrier density modulation in polymer field-effect transistors. Adv. Mater. 28, 57-62 (2016).

55. Sze, S. M. \& Ng, K. K. Physics of Semiconductor Devices. 3rd ed, (Wiley-Interscience, New Jersey, 2007).

56. Lin, Y. H., Faber, H., Rossbauer, S. \& Anthopoulos, T. D. Solution-processed ZnO nanoparticle-based transistors via a room-temperature photochemical conversion process. Appl. Phys. Lett. 102, 193516 (2013).

57. Horowitz, G. \& Delannoy, P. An analytical model for organic-based thin-film transistors. J. Appl. Phys. 70, 469-475 (1991).

58. Cochran, J. E. et al. Molecular interactions and ordering in electrically doped polymers: blends of PBTT and F 4 TCNQ. Macromolecules 47, 6836-6846 (2014).

59. Schlitz, R. A. et al. Solubility-limited extrinsic n-type doping of a high electron mobility polymer for thermoelectric applications. Adv. Mater. 26, 2825-2830 (2014).

(i) Open Access This article is licensed under a Creative Commons Attribution 4.0 International License, which permits use, sharing, adaptation, distribution and reproduction in any medium or format, as long as you give appropriate credit to the original author(s) and the source, provide a link to the Creative Commons license, and indicate if changes were made. The images or other third party material in this article are included in the article's Creative Commons license, unless indicated otherwise in a credit line to the material. If material is not included in the article's Creative Commons license and your intended use is not permitted by statutory regulation or exceeds the permitted use, you will need to obtain permission directly from the copyright holder. To view a copy of this license, visit http://creativecommons. org/licenses/by/4.0/.

(c) The Author(s) 2018 